\title{
Assessment of Wavelets Transform based Processing of Features of Forearm Muscle Signals for Prosthesis
}

\author{
M. Karuna, Sitaramanjaneya Reddy Guntur
}

\begin{abstract}
People who have lost forearm are suffer from hand mobility limitations due to trauma, disease or defect, Prosthesis arm help those people to do their daily actions. Researchers have been focused on developing artificial hand. In this regard, better processing of features of electromyographic (EMG) signal has a significant role from residual forearm muscle. To achieve this, Wavelet Transform (WT) technique has been applied because it is acceptable with the characteristics of EMG as a nonstationary signal. Results have shown that db5 wavelet decomposition performs best denoising at fifth level in other wavelets comparison. Furthermore, the ratio of Signal to Noise $(S / N)$ and the error of percentage (PE) are calculated to evaluate the eminence and the usefulness features of EMG.
\end{abstract}

Key Words: EMG, WT, Decomposition, Denoising, feature extraction, feature selection.

\section{INTRODUCTION}

EMG signal detected at the surface of the skin which determines the electrical current produced in fibres of muscle [1]. The main application of this research is to identify the various patterns of sEMG signal for controlling the prosthesis [2-3].

Noises are created in the EMG signal due to various sources such as the hardware for amplification, digital processing for analog to digital conversion and cables used for acquiring data as well as activity of motors at distance from detection area. Preprocessing of signal from muscle fibres acts an important role in realm of clinical and rehabilitation applications. Some methods to remove noise from the detected EMG signal have been emphasized by Cram et al. [4]. The major drawback of identifying the intentional movement is the inadequate consequences under the environment of presented noises, particularly when the random noise frequency characteristics. According to the literary sources, many researchers have suggested noise removal techniques from EMG signals by using digital filters [5].

Even though above filters can decrease the considerable noise, and also traces distortion in the EMG signal [6]. In recent research, the denoising WT theory is found very efficient in processing of denoise [7-10].

Therefore, signal decomposition, noise reduction from sEMG signal [11] using wavelets presented as shown in Fig.1. Moreover, an important requirement is to differentiate various EMG signals accurately for controlling prosthesis is effective extraction of features.

Revised Manuscript Received on December 15, 2019.

M. Karuna, Dept. of Electronics and Communication Engineering, Vignan Foundation for Science, Technology and Research, India.

Sitaramanjaneya Reddy Guntur, Dept. of Electronics and Communication Engineering, Vignan Foundation for Science, Technology and Research, India.drgsr_ece@ vignan.ac.in
The techniques based on the extraction of feature have been effectively used for recognising different forearm muscle movements [12].

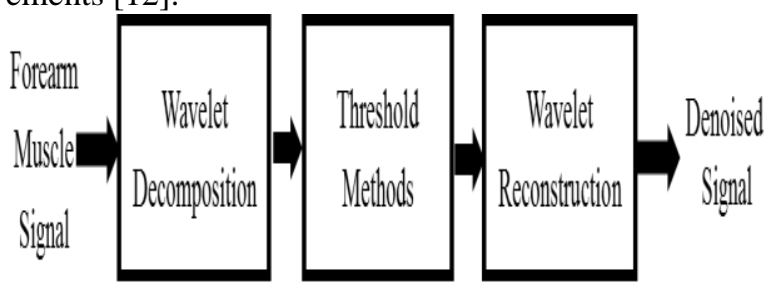

Fig.1 Block diagram of wavelet denoising process In the present work were examined the effectiveness of denoising forearm muscle signals, with Stationary wavelet transform (SWT) and db5 at fifth level of decomposition of EMG signal by calculating the $\mathrm{S} / \mathrm{N}$ values of the noise eliminated signals and Percentage Error (PE). In addition to that, a relative study was realized to picturize the efficiency of EMG features. The robustness of this approach depends on the better feature extraction.

\section{METHODOLOGY}

The four healthy male subjects were instructed to perform the wrist actions such as extension, flexion, pronation and supination. EMG detector used to collect EMG signals of forearm muscles, in which outputs for the signals, gain was adjusted to $60 \mathrm{~dB}$ and bandwidth is limited to $20 \mathrm{~Hz}-500 \mathrm{~Hz}$ with the help of main amplifier and filter.The sEMG signal was recorded by placing surface electrodes $(\mathrm{Ag}-\mathrm{AgCl})$ on the right forearm muscles such as flexor carpi radialis and extensor carpi radialis longus of a subject [13]. The equal distance of $2 \mathrm{~cm}$ is maintained between electrodes. One electrode is placed on the center of the muscle structure and other one is at the end. The third electrode was positioned on parts having no muscles on being bony. For each motion four datasets were collected. Recognitions of intentional movement through EMG signals have traced out markable results, since the last half a century period as a solution for dexterous prosthetic control to perform multifunctions. 


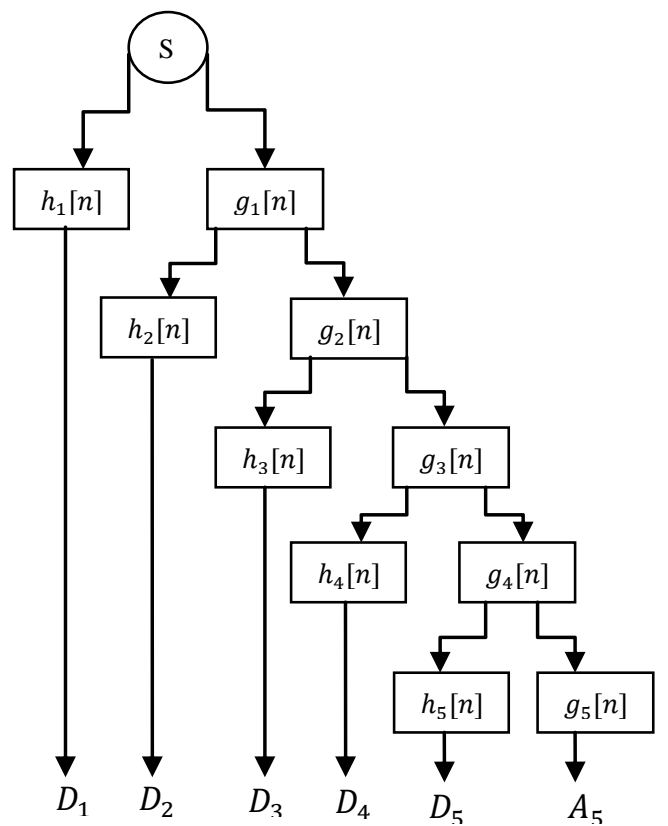

Fig.2 DWT decomposition with SWT filter bank scheme Fig.1 shows the approach of EMG pattern identification commonly fundamental processing stages were included. However, the passion rising within the scholastic group is associated by improper impact in clinical and commercial realm [14-15].

\section{A. Decomposition}

The initial step is to remove noise from raw EMG signal by applying WT decomposition and denoising methods using SWT. Significant sub-sampling makes DWT, the most effectively computed the wavelet transform. Nevertheless, variance to time shifts suffers at DWT. This might be without consequence because the perfect restoration ability of inverse DWT, however, it becomes unstable when translationinvariant signal investigation is performed in the WT coefficient subspace. SWT achieves translation-invariance by using unnecessary WT representation as the signal subsampling is removed in the DWT. Besides, the filter coefficients are up-sampled with doubling effect in each stage of processing. The fundamental principle is to take means of slightly different DWT, also known as $\varepsilon$-decimated DWT [16]. The signals with various resolution at various frequency bands are analyzed with the technique of decomposition of signal into approximations and the coefficients details are shown in Fig.2. The decomposition process involves different frequency bands in successive low-pass and high-pass filtering of the distinct time domain signal. In this study, Daubechie (db5) WT function was used at fifth level of decomposition.

\section{B. Denoising}

The detected EMG signals of forearm muscle were processed denoising, using the SWT and a threshold method. This is implemented with MATLAB wavelet toolbox to remove intervention of noise using the WT based denoising. The resulting SWT coefficients are thresholded by universal threshold method. Eventually, the accurate signal is retrieved by using the inverse wavelet transform, from the thresholded SWT coefficients. Therefore, retrieved signals, such as D1, D2, D3, D4, D5, and A5were extracted for calculating features (Fig 2).

\section{Feature extraction}

The EMG signals at the surface depends on time and amplitude changes randomly around zero and hence, the analysis of signal is necessary to define the salient properties. A number of features have been taken up both in group and separately for representing the cases of surface EMG amplitude and content of spectrum [17-19]. Because of the computational simplicity, time domain variables are determined as a function of time. In EMG signal identification the time domain features are most accepted. Root mean square (RMS), mean absolute value (MAV), variance (VAR) of EMG is used to test the performance. Later the evaluation criteria for finding the effectiveness property of the features are used. The factor determined in the process includes.

1. RMS: It is a statement factor deciding variation in magnitude [20], especially important for cases in which variants are either positive or negative, as variables are quantified by RMS value. Higher resolution implies high energy. It is defined as

$$
\mathrm{RMS}=\sqrt{\frac{1}{N} \sum_{m=1}^{N} x_{m}^{2}}
$$

2. MAV: It is an mean of absolute value of the EMG signal amplitude [21] and is defined as

$$
\mathrm{MAV}=\frac{1}{N} \sum_{m=1}^{N}\left|x_{m}\right|
$$

3. VAR: It is a measure of mean of square value of divergence of variable [22] and is defined as:

$$
\mathrm{VAR}=\frac{1}{N-1} \sum_{m=1}^{N} x_{m}^{2}
$$

\section{Evaluation criteria}

The ratio of SNR and PE are used as performance criteria to evaluate the efficiency and the robustness features of EMG [23].SNR can be computed using the formulae given by

$$
\mathrm{SNR}=10 \log \frac{P_{\text {denoise }}}{P_{\text {noise }}}
$$

Where, $P_{\text {denoise }}$ is the denoised sEMG signal power and $P_{\text {noise }}$ is the interference power of EMG signal.

$$
\mathrm{PE}=\frac{\mid \text { feature }_{\text {denoise }}-\text { feature }_{\text {noise }} \mid}{\text { feature }_{\text {denoise }}} \times 100 \%
$$

Where feature denoise a vector is represents features from denoising of sEMG signal and feature noise $_{\text {is a vector }}$ represents the features of the noisy sEMG signal.

\section{RESULTS AND DISCUSSION}

In this research, decomposition of the raw EMG signal as shown in Fig.3 using Daubechie (db5) at level five of decomposition as shown in Fig.4 and obtained coefficients details and denoised the forearm EMG signals using SWT denoising method and universal threshold method to the coefficient (d5) details, the obtained denoised EMG signal of forearm supination as appears in Fig.5.

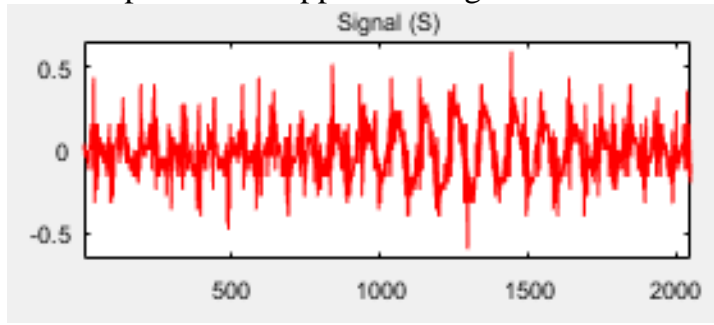

Fig.3. Raw EMG signal of forearm Supination. 


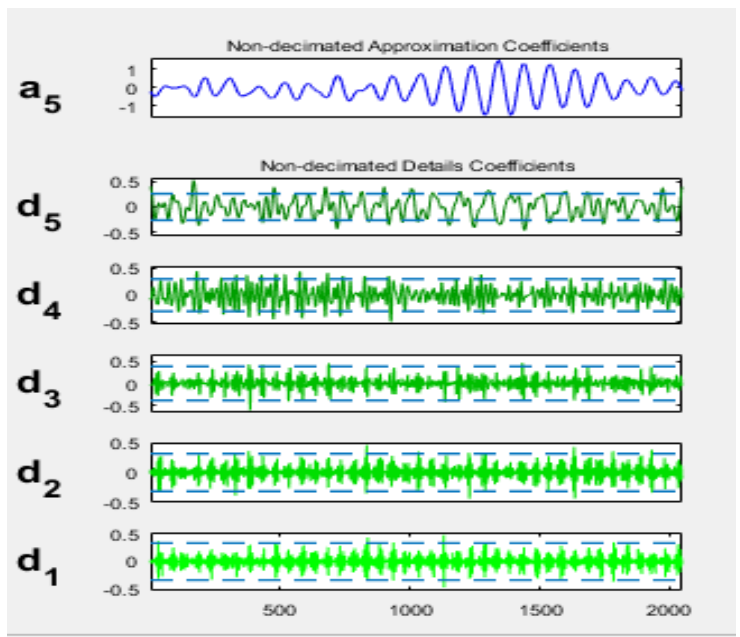

Fig.4. Decomposition of forearm EMG signal at $5^{\text {th }}$ level

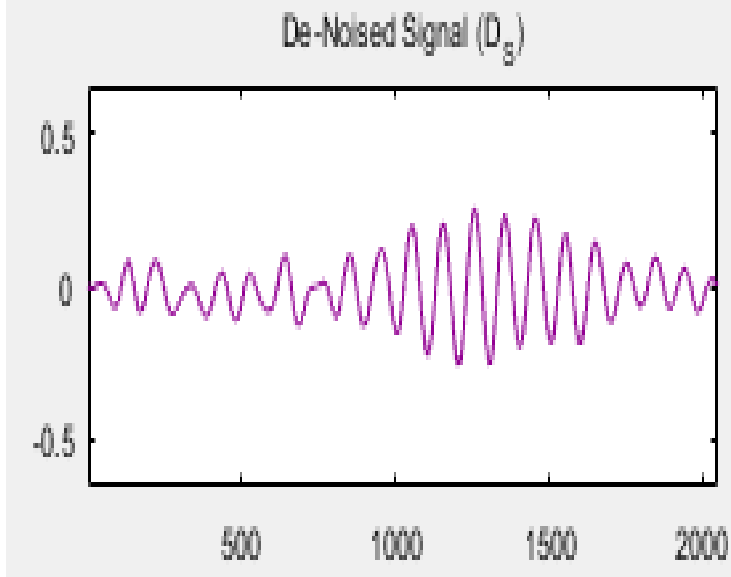

Fig.5 Denoised EMG signal of forearm Supination After wavelet based denoising of EMG signals, three features are extracted and calculated the SNR values, PE with sEMG signal. Fig. 6 shows the variation of PE against SNR values of the four subjects determines the effectiveness can be selected that is useful for application depends on the

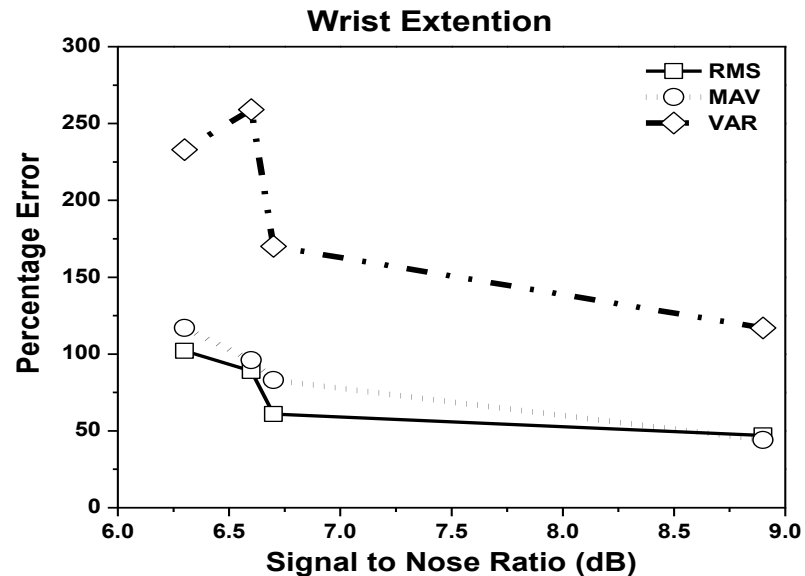

(a) of three time domain features, in practice extracted feature amount of interference of sEMG mechanism.

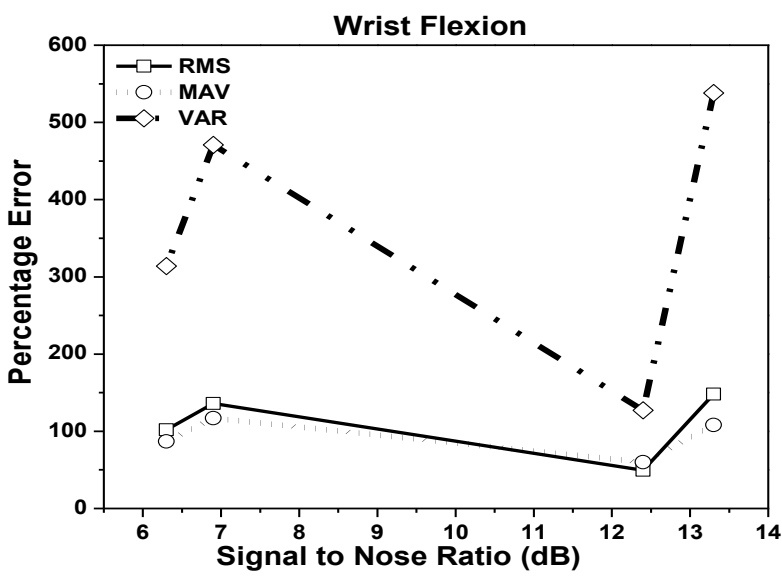

(b)

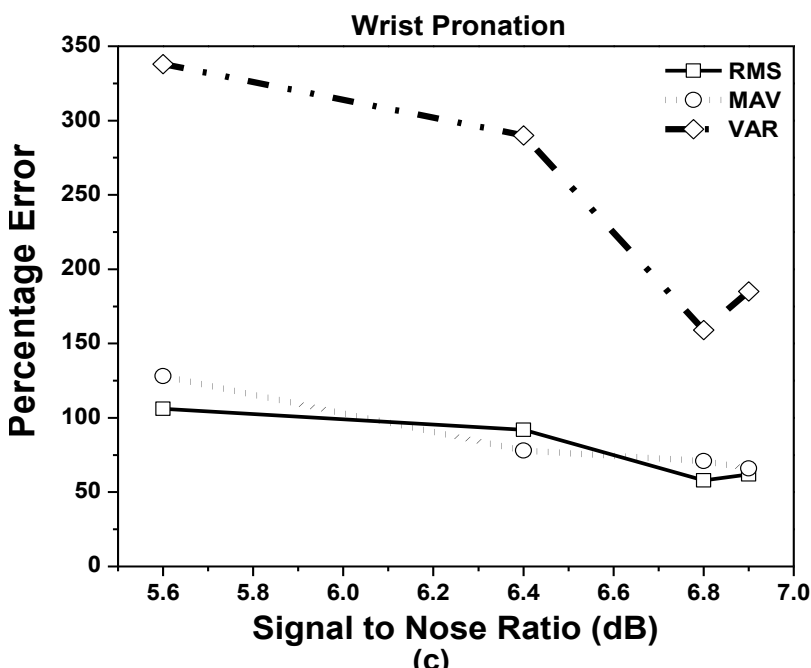

(c)

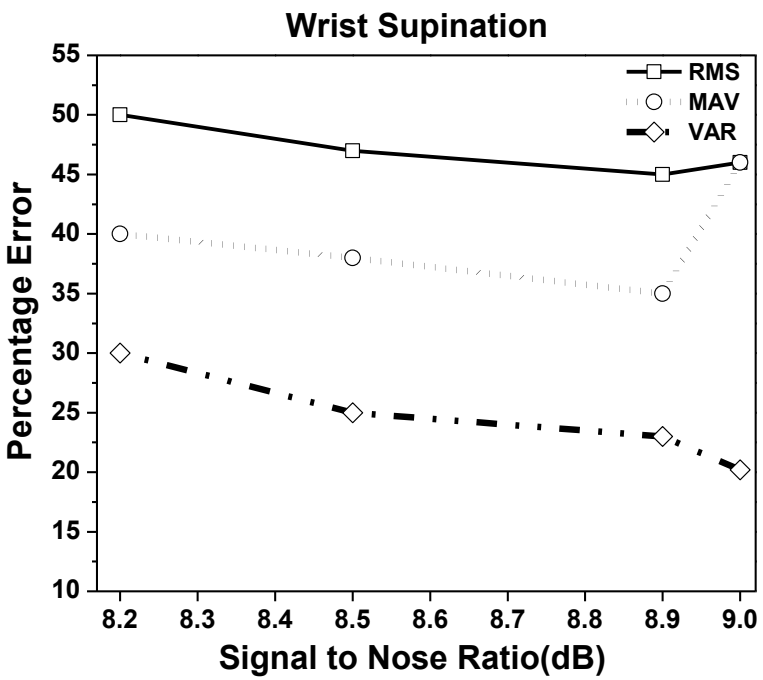

(d)

Fig.6. The percentage error of denoised forearm SEMG signals of selected RMS, MAV and VAR features at various signal to noise ratios $(\mathrm{dB})$ in four motions of wrist (a) Extention,(b) Flexion, (c)Pronation, and (d) Supination.

The smallest value of PE is considered as the best performance methods. The highest values of SNR showed for denoised sEMG signals, the lowest SNR is for noisy signals. Fig.6 (a, c) shows RMS feature of SNR value is $9 \mathrm{~dB}$ at lowest PE is better for wrist extention, wrist pronation and Fig.6(b) results show that the MAV and RMS are suitable 
features for wrist flexion also highest SNR is obtained than the other movements. Fig.6 (d) shows that VAR feature is having highest SNR with low PE for wrist supination. In recent studies, a novel method based on empirical mode decomposition (EMD) showed effective results in biomedical signal filtering. In future work the performance of the combination of the wavelet transform with the EMD technique is to be tested by extracting features and calculating performance evaluation of PE in forearm EMG signal denoising compared with each method alone.

\section{IV.CONCLUSION}

In present study, wavelet transform was successfully applied to forearm EMG signals denoising. Different features were calculated to get optimized features after performing denoising procedure at fifth level of decomposition using db5 wavelets. In this research obtained results are shown clearly that different features are suitable for different wrist movements from performance evaluation of SNR and PE. From the results of the comparative study, it may be concluded that the RMS feature is the best for wrist extension and pronation, flexion and VAR feature is suitable for identifying the wrist supination.

\section{REFERENCES}

1. Hudgins B, Parker P and Scott R.N. "A new strategy for multifunctional myoelectric control", IEEE Trans. On Biomed. Eng., 40, 1, 82-94, 1993.

2. Oskoei M.A. and $\mathrm{Hu}$ H. "Myoelectric control systems- A survey" Biomedical Signal Processing and Control, 2, 4, 275-294, 2007.

3. Parker P, Englehart K, Hudgins B. "Myoelectric signal processing for control of powered limb prostheses," Journal of Electromyography and Kinesiology, 16, 6, 541-548, 2006.

4. Cram J.R., Kasman G.S., Holtz J. "Introduction to surface electromyography", $1^{\text {st }}$ ed., Aspen Publishers, MD, USA, 1998.

5. Mewett D.T, Reynolds KJ, Nazeran H. "Reducing power line interference in digitised electromyogram recordings by spectrum interpolation", Med. Biol. Eng. Comput. 42, 524-531, 2004.

6. Clancy, E.A., Morin, E.L., Merletti, R. "Sampling, noise-reduction and amplitude estimation issues in surface electromyography". J. Electromyogr. Kinesiol. 12, 1-16, 2002.

7. Patil, P.B., Chavan, M.S., "A wavelet based method for denoising of biomedical signal”. ICPR, Informat. Med. Eng. 978-1-4673-10390/12, 2012.

8. Rabiul Islam, S.Md. Huang, X., Sharma, D. "Wavelet based denoising algorithm of the ECG signal corrupted by wgn and poisson noise," ISCIT. 978-1-4673-1157-1, 2012.

9. EI hanine, M., Abdelmounim, E., Haddadi, R.,Belaguid, A., "Electrocardiogram signal denoising using discrete wavelet transform", IEEE 978-1-4799-3824-7/14,2014.

10. AlMahamdy, M., Riley, H.B. "Performance study of different denoising methods for ECG signals". Procedia Comput. Sci. 37325 $-332,2014$

11. Merlo A and Farina D., "A fast and reliable technique for muscle activity detection from surface EMG signals," IEEE Trans. Biomed. Eng., 50, 316-323, 2003.

12. Englehart K., Hudgins B., Parker P, Stevenson M. "Improving myoelectric signal classification using wavelet packets and principle component analysis". Proc. of the $21^{\text {st }}$ Annual Int. Conf. of the IEEE on Eng. in Med. and Biol. Soc., Atlanta, 1999.

13. Merletti R and Parker P. "Electromyography physiology, engineering, and noninvasive applications. John Wiley Sons, Inc., New York, 2004

14. Atkins D.J, Heard D.C,Donovan W.H. "Epidemiologic overview of individuals with upper-limb loss and their reported research priorities", JPO: Journal of Prosthetics and Orthotics, Bd. 8, Nr. 1, 2 11, 1996.

15. Farina D and Aszmann O. "Bionic limbs: clinical reality and academic promises, "Science translational medicine, Bd. 6, Nr. 257, 257ps12, 2014.
16. Nason G.P and Silverman B.W, "The stationary wavelet transform and some statistical applications, 281-299 Hrsg., New York: Springer, 1995.

17. Micera Silvestro, "Control of hand prostheses using peripheral information", IEEE reviews in BME, 3, 48-68, 2010.

18. Englehart K, Hudgins B, Philip PA, Stevenson M, "Classification of the myoelectric signal using time-frequency based representations", Medical Engineering \& Physics, 21, 431-438, 1999.

19. Lawrence J. H. De Luca, "Myoelectric signal versus force relationship in different human muscles', Journal of Applied Physiology" 54, 1653-1659, 1983.

20. Boostani R and Moradi M.H, "Evaluation of the forearm EMG signal features for the control of a prosthetic hand", Journal of Physiological Measurement, 24,309-319, 2003.

21. Phinyomark A, Phukpattaranont P, and Limsakul C, "Feature reduction and selection for EMG signal classification". Expert Systems with Applications, 39, 8, 7420 - 7431, 2012.

22. Zardoshti-Kermani M, WheelerBC. "EMG feature evaluation for movement control of upper extremity prostheses". IEEE Transactions on rehabilitation engineering, 3, 324-333, 1995.

23. Phinyomark A, Limsakul C, Phukpattaranont P, "A Novel Feature extraction for robust EMG pattern recognition," Journal of Computing, 1, 70-80, 2009. 